\title{
Three-Step Two-Hybrid Block Method for the Direct Solution of Second-Order Ordinary Differential Equations
}

Raymond Dominic (Corresponding Author)

Department of Mathematics and Statistics, Federal University Wukari, Nigeria

Email: domehbazza@yahoo.co.uk

Kyagya Yusuf T.

Department of Mathematics and Statistics, Federal University Wukari, Nigeria

Article History

Received: February 3, 2020

Revised: February 27, 2020

Accepted: March 7, 2020

Published: March 9, 2020

Copyright (C) 2020 ARPG \&

Author

This work is licensed unde

the Creative Commons

Attribution International

(c) (1) mons Attribution License 4.0

\begin{abstract}
In this paper a three-step two hybrid block method with two offgrid hybrid points chosen within interval $\left[\mathrm{X}_{n}, \mathrm{X}_{\mathrm{n}+1}\right]$ and $\left[X_{n+1}, X_{n+2}\right]$ was developed to solve second Order Ordinary Differential Equations directly, using the power series as the basic function to approximate and generate some continuous schemes. The basic propertise of the method was investigated and was found to converge. Numerical Solution of our method was tested on some stiff equations and was found to give better approximation than the existing method.
\end{abstract}

Keywords: Three-step; Block method; Two hybrid points.

AMS Subject Classification: 65L05, 65L06, 65L20.

\section{Introduction}

In this paper we intend to solve second derivative initial value problem using power series of order fourteen given of the form

$$
y(x)=\sum_{j=0}^{2 s+r-1} a_{i}\left(\frac{x-x_{n}}{h}\right)^{j}
$$

which is proposed as general second derivative solution of initial value problems of the form

$$
y^{\prime \prime}=f\left(x, y(x), y^{\prime}(x)\right), y\left(x_{0}\right)=y_{0}, y^{\prime}\left(x_{0}\right)=y_{0}^{\prime}
$$

Solving higher order derivatives directly by block method tend to be better off than the reduction to system of first-order approach which involves more functions to evaluate. Its remedy the set back encounters in the reduction method, the direct block method retain some basic character of linear multistep method that share common propertise of Runge-Kutta at orther points order than the step points, as contain is Raymond, et al. [1], Raymond, et al. [2], Mohammad and Zurni [3], Abdelrahim and Zurni [4], Adeniran and Ogundare [5], Adesanya, et al. [6] to mentioned but a few. Also many researcher adopted the interpolation and collocation of power series to generate continuous linear multistep method, most especially Awoyemi, et al. [7] and Fatunla [8].

We proposed in this paper a three-step hybrid block third derivative method with two offgrid of order thirteen to solve some second order initial value problems directly which is implemented in block.

This paper is organise as follows: In section 2, we discuss the methods and the materials for the development of the method. Section 3 considers analysis of the basis properties of the method, which include convergence and stability region and some numerical experiments where the efficiency of the derived method is tested on some stiff numerical examples. Lastly, the conclusion shall be drawn in section 4.

\section{Derivation of the Method}

We intend to develop a method of the form

$$
y(x)=\sum_{j=0, \frac{1}{2}} \alpha_{j} y_{n+j}+\sum_{i=2}^{l} h^{i}\left[\sum_{j=0}^{3} \beta_{j} f_{n+j}+\beta_{k} f_{n+k}\right]+\sum_{i=3}^{l} h^{i}\left[\sum_{j=0}^{3} \gamma_{j} g_{n+j}+\gamma_{k} g_{n+k}\right] k=\frac{1}{2}, \frac{3}{2}
$$

Subject to the consistency condition

$$
\sum_{j=0}^{k} h^{i}\left(\beta_{j}+\beta_{k}\right)=0 \text {, where } k \text { is a rational number }
$$


$\beta_{j}(x), \beta_{k}(x), \gamma_{j}(x)$ and $\gamma_{k}(x)$ are the continuous coefficients of the method. ${ }^{y_{n+j}}$ the numerical approximation to the exact $\quad$ solution
$g_{n+j}=f^{\prime \prime \prime}\left(x_{n+j}, y\left(x_{n+j}\right)\right), j=0,1,2,3, k$

Consider the solution of the form

$$
y(x)=\sum_{j=0}^{2 s+r-1} a_{i}\left(\frac{x-x_{n}}{h}\right)^{j}
$$

where $r=2$ and $s=6$ are the numbers of interpolation and collocation points respectively, is considered to be a solution to (1).

Differentiating (2) twice and thrice gives

$$
\left.\begin{array}{l}
y^{\prime \prime}(x)=\sum_{j=2}^{2 s+r-1} \frac{a_{j} j !}{h^{2}(j-2) !}\left(\frac{x-x_{n}}{h}\right)^{j-2}=f\left(x, y, y^{\prime}\right) \\
y^{\prime \prime \prime}(x)=\sum_{j=3}^{2 s+r-1} \frac{a_{j} j !}{h^{2}(j-3) !}\left(\frac{x-x_{n}}{h}\right)^{j-3}=g\left(x, y, y^{\prime}, y^{\prime \prime}\right)
\end{array}\right\}
$$

Substituting (3) into (1) gives

$$
f\left(x, y, y^{\prime \prime}\right)=\sum_{j=2}^{2 s+r-1} \frac{a_{j} j !}{h^{2}(j-2) !}\left(\frac{x-x_{n}}{h}\right)^{j-2}+\sum_{j=3}^{2 s+r-1} \frac{a_{j} j !}{h^{2}(j-3) !}\left(\frac{x-x_{n}}{h}\right)^{j-3}
$$

Collocating (4) at all points $x_{n+s}, s=0, \frac{1}{2}, 1, \frac{3}{2}, 2,3$ and Interpolating Equation (2) at $x_{n+r}, r=0, \frac{1}{2}$, gives a system of non linear equation of the form

$$
\left.\begin{array}{l}
\sum_{j=0}^{2 s+r-1} \alpha_{j}\left(\frac{x-x_{n}}{h}\right)^{j}=y_{n+j} \\
\sum_{j=2}^{2 s+r-1} \frac{a_{j} j !}{h^{2}(j-2) !}\left(\frac{x-x_{n}}{h}\right)^{j-2}=f^{\prime}\left(x, y, y^{\prime}\right) \\
\sum_{j=2}^{2 s+r-1} \frac{a_{j} j !}{h^{2}(j-2) !}\left(\frac{x-x_{n}}{h}\right)^{j-3}=g^{\prime}\left(x, y, y^{\prime}, y^{\prime \prime}\right)
\end{array}\right\}
$$

Solving (5) for ${ }^{2}{ }^{\prime} s$ using Gaussian elimination method, gives a continuous hybrid linear multistep method of the form

$$
y(x)=\sum_{j=0, \frac{1}{2}} \alpha_{j} y_{n+j}+\sum_{i=2}^{l} h^{i}\left[\sum_{j=0}^{3} \beta_{j} f_{n+j}+\beta_{k} f_{n+k}\right]+\sum_{i=3}^{l} h^{i}\left[\sum_{j=0}^{3} \gamma_{j} g_{n+j}+\gamma_{k} g_{n+k}\right] k=\frac{1}{2}, \frac{3}{2}
$$

where

$$
\begin{aligned}
& \alpha_{0}=1-\frac{2\left(-x_{n}+x\right)}{h} \quad \alpha_{\frac{1}{2}}=\frac{2\left(-x_{n}+x\right)}{h} \\
& \beta_{0}=-\frac{3920185597}{29889699840}\left(x-x_{n}\right) h+\frac{1}{2}\left(x-x_{n}\right)^{2}-\frac{1667}{432} \frac{\left(x-x_{n}\right)^{4}}{h^{2}}+\frac{8521}{720} \frac{\left(x-x_{n}\right)^{5}}{h^{3}} \\
& -\frac{93439}{4860} \frac{\left(x-x_{n}\right)^{6}}{h^{4}} \\
& +\frac{135475}{6804} \frac{\left(x-x_{n}\right)^{7}}{h^{5}}-\frac{36037}{2592} \frac{\left(x-x_{n}\right)^{8}}{h^{6}}+\frac{154817}{23328} \frac{\left(x-x_{n}\right)^{9}}{h^{7}}-\frac{7787}{3645} \frac{\left(x-x_{n}\right)^{10}}{h^{8}} \\
& +\frac{395}{891} \frac{\left(x-x_{n}\right)^{11}}{h^{9}}
\end{aligned}
$$


$-\frac{13}{243} \frac{\left(x-x_{n}\right)^{12}}{h^{10}}+\frac{1}{351} \frac{\left(x-x_{n}\right)^{13}}{h^{11}}$

$\beta_{\frac{1}{2}}=\frac{93222191}{4864860000}\left(x-x_{n}\right) h-\frac{1024}{125} \frac{\left(x-x_{n}\right)^{4}}{h^{2}}+\frac{27264}{625} \frac{\left(x-x_{n}\right)^{5}}{h^{3}}-\frac{110848}{1125} \frac{\left(x-x_{n}\right)^{6}}{h^{4}}$

$+\frac{22208}{175} \frac{\left(x-x_{n}\right)^{7}}{h^{5}}-\frac{348608}{3375} \frac{\left(x-x_{n}\right)^{8}}{h^{6}}+\frac{1675552}{30375} \frac{\left(x-x_{n}\right)^{9}}{h^{7}}-\frac{587008}{30375} \frac{\left(x-x_{n}\right)^{10}}{h^{8}}$

$+\frac{158912}{37125} \frac{\left(x-x_{n}\right)^{11}}{h^{9}}-\frac{5504}{10125} \frac{\left(x-x_{n}\right)^{12}}{h^{10}}+\frac{3968}{131625} \frac{\left(x-x_{n}\right)^{13}}{h^{11}}$

$\beta_{1}=-\frac{6166247}{369008640}\left(x-x_{n}\right) h+\frac{81}{20} \frac{\left(x-x_{n}\right)^{5}}{h^{3}}-\frac{189}{10} \frac{\left(x-x_{n}\right)^{6}}{h^{4}}+\frac{2127}{56} \frac{\left(x-x_{n}\right)^{7}}{h^{5}}-\frac{675}{16} \frac{\left(x-x_{n}\right)^{8}}{h^{6}}$

$+\frac{8161}{288} \frac{\left(x-x_{n}\right)^{9}}{h^{7}}-\frac{532}{45} \frac{\left(x-x_{n}\right)^{10}}{h^{8}}+\frac{3\left(x-x_{n}\right)^{11}}{h^{9}}-\frac{14}{33} \frac{\left(x-x_{n}\right)^{12}}{h^{10}}+\frac{1}{39} \frac{\left(x-x_{n}\right)^{13}}{h^{11}}$

$\beta_{\frac{3}{2}}=-\frac{11284457}{116756640}\left(x-x_{n}\right) h+\frac{256}{27} \frac{\left(x-x_{n}\right)^{4}}{h^{2}}-\frac{6272}{135} \frac{\left(x-x_{n}\right)^{5}}{h^{3}}+\frac{128384}{1215} \frac{\left(x-x_{n}\right)^{6}}{h^{4}}$

$-\frac{8896}{63} \frac{\left(x-x_{n}\right)^{7}}{h^{5}}$

$+\frac{9728}{81} \frac{\left(x-x_{n}\right)^{8}}{h^{6}}-\frac{48736}{729} \frac{\left(x-x_{n}\right)^{9}}{h^{7}}+\frac{88448}{3645} \frac{\left(x-x_{n}\right)^{10}}{h^{8}}-\frac{448}{81} \frac{\left(x-x_{n}\right)^{11}}{h^{9}}+\frac{640}{891} \frac{\left(x-x_{n}\right)^{12}}{h^{10}}$ $-\frac{128}{3159} \frac{\left(x-x_{n}\right)^{13}}{h^{11}}$

$\beta_{2}=-\frac{244941409}{9963233280}\left(x-x_{n}\right) h+\frac{41}{16} \frac{\left(x-x_{n}\right)^{4}}{h^{2}}-\frac{1041}{80} \frac{\left(x-x_{n}\right)^{5}}{h^{3}}+\frac{5563}{180} \frac{\left(x-x_{n}\right)^{6}}{h^{4}}$ $-\frac{1217}{28} \frac{\left(x-x_{n}\right)^{7}}{h^{5}}$

$+\frac{33839}{864} \frac{\left(x-x_{n}\right)^{8}}{h^{6}}-\frac{180475}{7776} \frac{\left(x-x_{n}\right)^{9}}{h^{7}}+\frac{10921}{1215} \frac{\left(x-x_{n}\right)^{10}}{h^{8}}-\frac{59}{27} \frac{\left(-x_{n}\right)^{11}}{h^{9}}+\frac{269}{891} \frac{\left(x-x_{n}\right)^{12}}{h^{10}}$ $-\frac{19}{1053} \frac{\left(x-x_{n}\right)^{13}}{h^{11}}$

$\beta_{3}=-\frac{78553271}{1245404160000}\left(x-x_{n}\right) h+\frac{23}{3375} \frac{\left(x-x_{n}\right)^{4}}{h^{2}}-\frac{2387}{67500} \frac{\left(x-x_{n}\right)^{5}}{h^{3}}+\frac{2621}{30375} \frac{\left(x-x_{n}\right)^{6}}{h^{4}}$ $-\frac{42677}{340200} \frac{\left(x-x_{n}\right)^{7}}{h^{5}}+\frac{6353}{54000} \frac{\left(x-x_{n}\right)^{8}}{h^{6}}-\frac{71039}{972000} \frac{\left(x-x_{n}\right)^{9}}{h^{7}}$

$+\frac{908}{30375} \frac{\left(x-x_{n}\right)^{10}}{h^{8}}-\frac{287}{37125} \frac{\left(x-x_{n}\right)^{11}}{h^{9}}$

$+\frac{382}{334125} \frac{\left(x-x_{n}\right)^{12}}{h^{10}}-\frac{29}{394875} \frac{\left(x-x_{n}\right)^{13}}{h^{11}}$

$\gamma_{0}=-\frac{169905979}{29889699840}\left(x-x_{n}\right) h^{2}+\frac{1}{6}\left(x-x_{n}\right)^{3}-\frac{3}{4} \frac{\left(x-x_{n}\right)^{4}}{h}+\frac{1249}{720} \frac{\left(x-x_{n}\right)^{5}}{h^{2}}-\frac{68}{27} \frac{\left(x-x_{n}\right)^{6}}{h^{3}}$

$+\frac{16721}{6804} \frac{\left(x-x_{n}\right)^{7}}{h^{4}}-\frac{7507}{4536} \frac{\left(x-x_{n}\right)^{8}}{h^{5}}+\frac{17993}{23328} \frac{\left(x-x_{n}\right)^{9}}{h^{6}}-\frac{178}{729} \frac{\left(x-x_{n}\right)^{10}}{h^{7}}+\frac{223}{4455} \frac{\left(x-x_{n}\right)^{11}}{h^{8}}$

$-\frac{16}{2673} \frac{\left(x-x_{n}\right)^{12}}{h^{9}}+\frac{1}{3159} \frac{\left(x-x_{n}\right)^{13}}{h^{10}}$ 


$$
\begin{aligned}
& \gamma_{\frac{1}{2}}=\frac{8968109}{162162000}\left(x-x_{n}\right) h^{2}-\frac{96}{25} \frac{\left(x-x_{n}\right)^{4}}{h}+\frac{2016}{125} \frac{\left(x-x_{n}\right)^{5}}{h^{2}}-\frac{2384}{75} \frac{\left(x-x_{n}\right)^{6}}{h^{3}} \\
& +\frac{1312}{35} \frac{\left(x-x_{n}\right)^{7}}{h^{4}} \\
& -\frac{45208}{1575} \frac{\left(x-x_{n}\right)^{8}}{h^{5}}+\frac{29696}{2025} \frac{\left(x-x_{n}\right)^{9}}{h^{6}}-\frac{10064}{2025} \frac{\left(x-x_{n}\right)^{10}}{h^{7}}+\frac{2656}{2475} \frac{\left(x-x_{n}\right)^{11}}{h^{8}} \\
& -\frac{992}{7425} \frac{\left(x-x_{n}\right)^{12}}{h^{9}} \\
& +\frac{64}{8775} \frac{\left(x-x_{n}\right)^{13}}{h^{10}} \\
& \gamma_{1}=\frac{3772517}{52715520}\left(x-x_{n}\right) h^{2}-\frac{27}{4} \frac{\left(x-x_{n}\right)^{4}}{h}+\frac{162}{5} \frac{\left(x-x_{n}\right)^{5}}{h^{2}}-\frac{2883}{40} \frac{\left(x-x_{n}\right)^{6}}{h^{3}}+\frac{5277}{56} \frac{\left(x-x_{n}\right)^{7}}{h^{4}} \\
& -\frac{17611}{224} \frac{\left(x-x_{n}\right)^{8}}{h^{5}}+\frac{4139}{96} \frac{\left(x-x_{n}\right)^{9}}{h^{6}}-\frac{697}{45} \frac{\left(x-x_{n}\right)^{10}}{h^{7}}+\frac{193}{55} \frac{\left(x-x_{n}\right)^{11}}{h^{8}}-\frac{5}{11} \frac{\left(x-x_{n}\right)^{12}}{h^{9}} \\
& +\frac{1}{39} \frac{\left(x-x_{n}\right)^{13}}{h^{10}} \\
& \gamma_{\frac{3}{2}}=\frac{4089983}{116756640}\left(x-x_{n}\right) h^{2}-\frac{32}{9} \frac{\left(x-x_{n}\right)^{4}}{h}+\frac{160}{9} \frac{\left(x-x_{n}\right)^{5}}{h^{2}}-\frac{16784}{405} \frac{\left(x-x_{n}\right)^{6}}{h^{3}} \\
& +\frac{13856}{243} \frac{\left(x-x_{n}\right)^{7}}{h^{4}} \\
& -\frac{28424}{567} \frac{\left(x-x_{n}\right)^{8}}{h^{5}}+\frac{21088}{729} \frac{\left(x-x_{n}\right)^{9}}{h^{6}}-\frac{39728}{3645} \frac{\left(x-x_{n}\right)^{10}}{h^{7}}+\frac{11488}{4455} \frac{\left(x-x_{n}\right)^{11}}{h^{8}} \\
& -\frac{928}{2673} \frac{\left(x-x_{n}\right)^{12}}{h^{9}} \\
& +\frac{64}{3159} \frac{\left(x-x_{n}\right)^{13}}{h^{10}} \\
& \gamma_{2}=\frac{11862673}{3321077760}\left(x-x_{n}\right) h^{2}-\frac{3}{8} \frac{\left(x-x_{n}\right)^{4}}{h}+\frac{153}{80} \frac{\left(x-x_{n}\right)^{5}}{h^{2}}-\frac{137}{30} \frac{\left(x-x_{n}\right)^{6}}{h^{3}}+\frac{181}{28} \frac{\left(x-x_{n}\right)^{7}}{h^{4}} \\
& -\frac{5917}{1008} \frac{\left(x-x_{n}\right)^{8}}{h^{5}}+\frac{9097}{2592} \frac{\left(x-x_{n}\right)^{9}}{h^{6}}-\frac{556}{405} \frac{\left(x-x_{n}\right)^{10}}{h^{7}}+\frac{167}{495} \frac{\left(x-x_{n}\right)^{11}}{h^{8}}-\frac{14}{297} \frac{\left(x-x_{n}\right)^{12}}{h^{9}} \\
& +\frac{1}{351} \frac{\left(x-x_{n}\right)^{13}}{h^{10}} \\
& \gamma_{3}=\frac{7658447}{747242496000}\left(x-x_{n}\right) h^{2}-\frac{1}{900} \frac{\left(x-x_{n}\right)^{4}}{h}+\frac{13}{2250} \frac{\left(x-x_{n}\right)^{5}}{h^{2}}-\frac{229}{16200} \frac{\left(x-x_{n}\right)^{6}}{h^{3}} \\
& +\frac{1403}{68040} \frac{\left(x-x_{n}\right)^{7}}{h^{4}}-\frac{8809}{453600} \frac{\left(x-x_{n}\right)^{8}}{h^{5}}+\frac{7073}{583200} \frac{\left(x-x_{n}\right)^{9}}{h^{6}} \\
& -\frac{91}{18225} \frac{\left(x-x_{n}\right)^{10}}{h^{7}}+\frac{29}{22275} \frac{\left(x-x_{n}\right)^{11}}{h^{8}} \\
& -\frac{13}{66825} \frac{\left(x-x_{n}\right)^{12}}{h^{9}}+\frac{1}{78975} \frac{\left(x-x_{n}\right)^{13}}{h^{10}}
\end{aligned}
$$

Differentiating (6) once yields

$$
p^{\prime}(x)=\frac{1}{h} \sum_{j=0, \frac{1}{2}} \alpha_{j} y_{n+j}+h\left[\sum_{j=\frac{1}{2}, \frac{3}{2}} \beta_{j} f_{n+j}+\sum_{j=0}^{3} \beta_{j} f_{n+j}\right]+h^{2}\left[\sum_{j=\frac{1}{2}, \frac{3}{2}} \beta_{j} f_{n+j}+\sum_{j=0}^{3} \beta_{j} f_{n+j}\right]
$$


Equation (6) is evaluated at the non-interpolating points $\left\{x_{n+1}, x_{n+\frac{3}{2}}, x_{n+2}, x_{n+3}\right\}$ and (7) at all points $\left\{x_{n}, x_{n+\frac{1}{2}}, x_{n+1}, x_{n+\frac{3}{2}}, x_{n+2}, x_{n+3}\right\}$, which produces the following general equations in block form
$A^{(0)} Y_{m}^{(i)}=\sum_{i=0}^{1} h^{i} e_{i} y_{n}^{i}+h^{2} b_{i} f\left(y_{n}\right)+h^{2} d_{i} f\left(y_{m}\right)+h^{3} c_{i} f\left(y_{n}\right)+h^{3} r_{i} f\left(y_{m}\right)$

Where

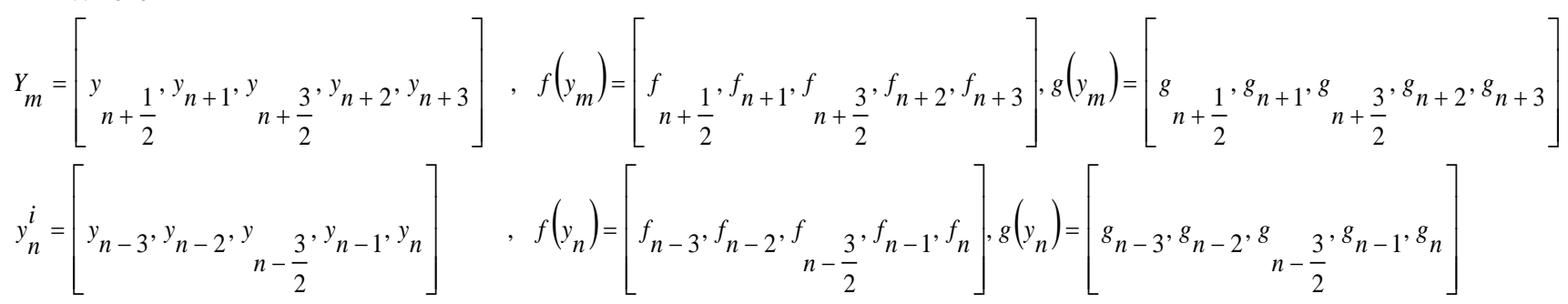
$e_{0}=\left[\begin{array}{lllll}0 & 0 & 0 & 0 & 1 \\ 0 & 0 & 0 & 0 & 1 \\ 0 & 0 & 0 & 0 & 1 \\ 0 & 0 & 0 & 0 & 1 \\ 0 & 0 & 0 & 0 & 1\end{array}\right], e_{1}=\left[\begin{array}{lllll}0 & 0 \times 10 & \text { identity matrix } \\ 0 & 0 & 0 & 0 & \frac{1}{2} \\ 0 & 0 & 0 & 0 & 1 \\ 0 & 0 & 0 & 0 & \frac{3}{2} \\ 0 & 0 & 0 & 0 & 2 \\ 0 & 0 & 0 & 0 & 3\end{array}\right], b_{0}=\left[\begin{array}{llllc}0 & 0 & 0 & 0 & \frac{3920185597}{5977939968} \\ 0 & 0 & 0 & 0 & \frac{1464961}{9729720} \\ 0 & 0 & 0 & 0 & \frac{19360779}{82001920} \\ 0 & 0 & 0 & 0 & \frac{1175047}{3648645} \\ 0 & 0 & 0 & 0 & \frac{52917}{80080}\end{array}\right], c_{0}=\left[\begin{array}{llllc}0 & 0 & 0 & 0 & \frac{169905979}{5977939968} \\ 0 & 0 & 0 & 0 & \frac{89963}{12972960} \\ 0 & 0 & 0 & 0 & \frac{129807}{11714560} \\ 0 & 0 & 0 & 0 & \frac{55714}{3648645} \\ 0 & 0 & 0 & 0 & \frac{603}{14560}\end{array}\right]$

$\begin{aligned} & d_{0}=\left[\begin{array}{ccccc}\frac{-93222191}{9729720000} & \frac{6166247}{738017280} & \frac{11284457}{233513280} & \frac{244941409}{1992646650} & \frac{78553271}{249080832000} \\ \frac{5791264}{50675625} & \frac{29129}{480480} & \frac{169952}{1216215} & \frac{112547}{3243240} & \frac{428531}{4864860000} \\ \frac{10804509}{40040000} & \frac{23372469}{82001920} & \frac{87639}{320320} & \frac{4943349}{82001920} & \frac{1543071}{102502400 \emptyset} \\ \frac{65818624}{152026875} & \frac{25112}{45045} & \frac{2097152}{3648645} & \frac{136849}{1216215} & \frac{34376}{152026875} \\ \frac{1946592}{625625} & \frac{802629}{160160} & -\frac{7968}{5005} & -\frac{219753}{80080} & \frac{1051431}{20020000}\end{array}\right], \\ & r_{0}=\left[\begin{array}{cccccc}\frac{-8968109}{324324000} & \frac{-3772517}{105431040} & \frac{-4089983}{233513280} & \frac{-11862673}{6642155520} & \frac{-7658447}{1494484991000} \\ \frac{-36104}{482625} & \frac{-38743}{360360} & \frac{-20248}{405405} & \frac{-2417}{480480} & \frac{-773}{54054000} \\ \frac{-948429}{8008000} & \frac{-15009381}{82001920} & \frac{-14373}{160160} & \frac{-64881}{7454720} & \frac{-50049}{2050048000} \\ \frac{-147968}{921375} & \frac{-1012}{4095} & \frac{-88576}{729729} & \frac{-5932}{405405} & \frac{-476}{13030875} \\ \frac{33048}{125125} & \frac{159651}{80080} & \frac{13032}{5005} & \frac{17253}{22880} & \frac{-5391}{1001000}\end{array}\right],\end{aligned}$

when $\mathrm{i}=1$ 
Academic Journal of Applied Mathematical Sciences

$$
\begin{aligned}
& e_{0}=\left[\begin{array}{lllll}
0 & 0 & 0 & 0 & 1 \\
0 & 0 & 0 & 0 & 1 \\
0 & 0 & 0 & 0 & 1 \\
0 & 0 & 0 & 0 & 1 \\
0 & 0 & 0 & 0 & 1
\end{array}\right], b_{0}=\left[\begin{array}{ccccc}
0 & 0 & 0 & 0 & \frac{5044147}{29859840} \\
0 & 0 & 0 & 0 & \frac{19919}{116640} \\
0 & 0 & 0 & 0 & \frac{7019}{40960} \\
0 & 0 & 0 & 0 & \frac{629}{3645} \\
0 & 0 & 0 & 0 & \frac{103}{160}
\end{array}\right], d_{0}=\left[\begin{array}{cccccc}
\frac{87839}{972000} & \frac{140257}{4055040} & \frac{211177}{1283040} & \frac{4534709}{109486080} & \frac{96367}{912384000} \\
\frac{9248}{30375} & \frac{4379}{15840} & \frac{8032}{40095} & \frac{20473}{427680} & \frac{1279}{10692000} \\
\frac{1269}{4000} & \frac{234009}{450560} & \frac{769}{1760} & \frac{24759}{450560} & \frac{1471}{11264000} \\
\frac{2048}{6075} & \frac{284}{495} & \frac{26624}{40095} & \frac{3373}{13365} & \frac{4}{22275} \\
\frac{864}{125} & \frac{19683}{1760} & -\frac{416}{55} & -\frac{14877}{1760} & \frac{12197}{44000}
\end{array}\right] \\
& , c_{0}=\left[\begin{array}{ccccc}
0 & 0 & 0 & 0 & \frac{18588527}{2299207680} \\
0 & 0 & 0 & 0 & \frac{74203}{8981280} \\
0 & 0 & 0 & 0 & \frac{26247}{3153920} \\
0 & 0 & 0 & 0 & \frac{2371}{280665} \\
0 & 0 & 0 & 0 & \frac{723}{12320}
\end{array}\right] r_{0}=\left[\begin{array}{cccccc}
\frac{-1044947}{9979200} & \frac{-3487481}{28385280} & \frac{-213019}{3592512} & \frac{-1535441}{255467520} & \frac{-197189}{149603840} \\
\frac{-13792}{155925} & \frac{-18643}{110880} & \frac{-19648}{280665} & \frac{-6901}{997920} & \frac{-871}{44906400} \\
\frac{-40641}{123200} & \frac{-438129}{3153920} & \frac{-2319}{24640} & \frac{-4941}{630781} & \frac{-333}{15769600} \\
\frac{-512}{6237} & \frac{-80}{693} & \frac{-9728}{280665} & \frac{-619}{31185} & \frac{-8}{280665} \\
\frac{2592}{1925} & \frac{79461}{12320} & \frac{576}{77} & \frac{24219}{12320} & \frac{-1311}{61600}
\end{array}\right],
\end{aligned}
$$

\section{Analysis of Basic Properties of the Method}

\subsection{Order of the Block}

The linear operator associated with the block $(8)$ is defined as,

$$
L\{y(x) ; h\}=A^{(0)} Y_{m}^{(i)}-\sum_{i=0}^{1} h^{i} e_{i} y_{n}^{i}-h^{2}\left(b_{i} f\left(y_{n}\right)+d_{i} f\left(y_{m}\right)\right)-h^{3}\left(c_{i} g\left(y_{n}\right)+r_{i} g\left(y_{m}\right)\right)
$$

Expanding (9) using Taylor series and comparing the coefficients in $\mathrm{h}$ gives

$$
L\{y(x) ; h\}=c_{0} y(x)+c_{1} h y^{\prime}(x)+c_{2} h^{2} y^{\prime \prime}(x)+\ldots+c_{p} h^{p} y^{p}(x)+c_{p+1} h^{p+1} y^{p+1}(x)+c_{p+2} h^{p+2} y^{p+2}(x) \ldots
$$

Definition 1: the block (8) associated with the linear operator is said to be of order $p$ if $c_{0}=c_{1}=c_{2}=\ldots=c_{p}=0 \quad c_{p+1}=0$. and $c_{p+2} \neq 0, c_{p+2}$ is called the error constant and the local truncation error is given by

$$
t_{n+k}=c_{p+2} h^{(p+2)} y^{(p+2)}\left(x_{n}\right)+o\left(h^{p+3}\right)
$$

For our method

Comparing the coefficient of $h$ gives $C_{0}=C_{1}=C_{2}=C_{3}=\ldots=C_{13}=0$ and

$$
C_{14}=\left[\begin{array}{c}
\frac{10818347}{707022915895296000}, \frac{19391}{460301377536000}, \frac{22941}{323284369408000}, \frac{443}{4315325414400}, \frac{945}{22528}, \\
\frac{8968109}{176755728973824000}, \frac{38743}{690452066304000}, \frac{4791}{80821092352000}, \frac{1483}{21576627072000}, \frac{1797}{315707392000}
\end{array}\right]^{T}
$$

\subsection{Zero Stability of Our Method}

Definition 2: A block (8) is said to be zero-stable if as $h \rightarrow 0$, the root $z_{i}, i=1(1) k$ of the first characteristic polynomial $\rho(z)=0$ that is $\rho(z)=\operatorname{det}\left[\sum_{j=0}^{k} A^{(i)} z^{k-i}\right]=0 \quad$ Satisfies $\left|z_{i}\right| \leq 1$ and for those roots with $\left|z_{i}\right|=1$, multiplicity must not exceed two. The block method for $\mathrm{k}=3$, with two off-grid collocation point expressed in the form 


$$
\begin{aligned}
& \rho(z)=\left[\begin{array}{llllllllll}
1 & 0 & 0 & 0 & 0 & 0 & 0 & 0 & 0 & 0 \\
0 & 1 & 0 & 0 & 0 & 0 & 0 & 0 & 0 & 0 \\
0 & 0 & 1 & 0 & 0 & 0 & 0 & 0 & 0 & 0 \\
0 & 0 & 0 & 1 & 0 & 0 & 0 & 0 & 0 & 0 \\
0 & 0 & 0 & 0 & 1 & 0 & 0 & 0 & 0 & 0 \\
0 & 0 & 0 & 0 & 0 & 1 & 0 & 0 & 0 & 0 \\
0 & 0 & 0 & 0 & 0 & 0 & 1 & 0 & 0 & 0 \\
0 & 0 & 0 & 0 & 0 & 0 & 0 & 1 & 0 & 0 \\
0 & 0 & 0 & 0 & 0 & 0 & 0 & 0 & 1 & 0 \\
0 & 0 & 0 & 0 & 0 & 0 & 0 & 0 & 0 & 1
\end{array}\right]-\left[\begin{array}{lllllllllc}
0 & 0 & 0 & 0 & 1 & 0 & 0 & 0 & 0 & \frac{h}{2} \\
0 & 0 & 0 & 0 & 1 & 0 & 0 & 0 & 0 & h \\
0 & 0 & 0 & 0 & 1 & 0 & 0 & 0 & 0 & \frac{3 h}{2} \\
0 & 0 & 0 & 0 & 1 & 0 & 0 & 0 & 0 & 2 h \\
0 & 0 & 0 & 0 & 1 & 0 & 0 & 0 & 0 & 3 h \\
0 & 0 & 0 & 0 & 0 & 0 & 0 & 0 & 0 & 1 \\
0 & 0 & 0 & 0 & 0 & 0 & 0 & 0 & 0 & 1 \\
0 & 0 & 0 & 0 & 0 & 0 & 0 & 0 & 0 & 1 \\
0 & 0 & 0 & 0 & 0 & 0 & 0 & 0 & 0 & 1 \\
0 & 0 & 0 & 0 & 0 & 0 & 0 & 0 & 0 & 1
\end{array}\right]=z^{8}(z-1)^{2} \\
& \rho(z)=z^{8}(z-1)^{2}=0, \\
&
\end{aligned}
$$

Hence, our method is zero-stable.

\subsection{Regions of Absolute Stability (RAS)}

The stability polynomial for $\mathrm{K}=3$ with two off-step points gives

$$
\begin{aligned}
& \bar{h}(w)=-h^{15}\left(\left(\frac{752159}{454618644480000}\right) w^{4}-\left(\frac{367}{8742666240000}\right) w^{5}\right)-h^{14}\left(\left(\frac{1621}{11271536640000}\right) w^{5}+\left(\frac{970472249}{20457839001600000}\right) w^{4}\right) \\
& -h^{13}\left(\left(\frac{1711}{14529715200000}\right) w^{5}+\left(\frac{101922553}{227309322240000}\right) w^{4}\right)-h^{12}\left(\left(\frac{15052931}{20457839001600000}\right) w^{5}+\left(\frac{568986294211}{61373517004800000}\right) w^{4}\right) \\
& +h^{11}\left(\left(\frac{251133577241}{5260587171840000}\right) w^{4}-\left(\frac{55674431}{469695283200000}\right) w^{5}\right)-h^{10}\left(\left(\frac{1365924863}{1315146792960000}\right) w^{5}+\left(\frac{23620065761}{101165137920000}\right) w^{4}\right) \\
& +h^{9}\left(\left(\frac{86906999}{35227146240000}\right) w^{5}+\left(\frac{669035398667}{70454292480000}\right) w^{4}\right)+h^{8}\left(\left(\frac{22630147597}{197272018944000}\right) w^{5}+\left(\frac{62079338540911}{98636009472000}\right) w^{4}\right) \\
& -h^{7}\left(\left(\frac{756356329}{733898880000}\right) w^{5}+\left(\frac{125448836357}{287400960000}\right) w^{4}\right)+h^{6}\left(\left(\frac{116477849}{25369344000}\right) w^{5}+\left(\frac{39440548429}{25369344000}\right) w^{3}\right) \\
& -h^{5}\left(\left(\frac{2953}{449280}\right) w^{5}+\left(\frac{89987159}{19008000}\right) w^{4}\right)-h^{4}\left(\left(\frac{29613499}{741312000}\right) w^{5}-\left(\frac{4738380307}{741312000}\right) w^{3}\right)+h^{3}\left(\left(\frac{105077}{17820}\right) w^{4}-\left(\frac{5673887}{23166000}\right) w^{5}\right) \\
& -h^{2}\left(\left(\frac{4537583}{9266400}\right) w^{5}+\left(\frac{37161217}{9266400}\right) w^{3}\right)+w^{5}-w^{4}
\end{aligned}
$$

The absolute stability region of our method is then given by figure below

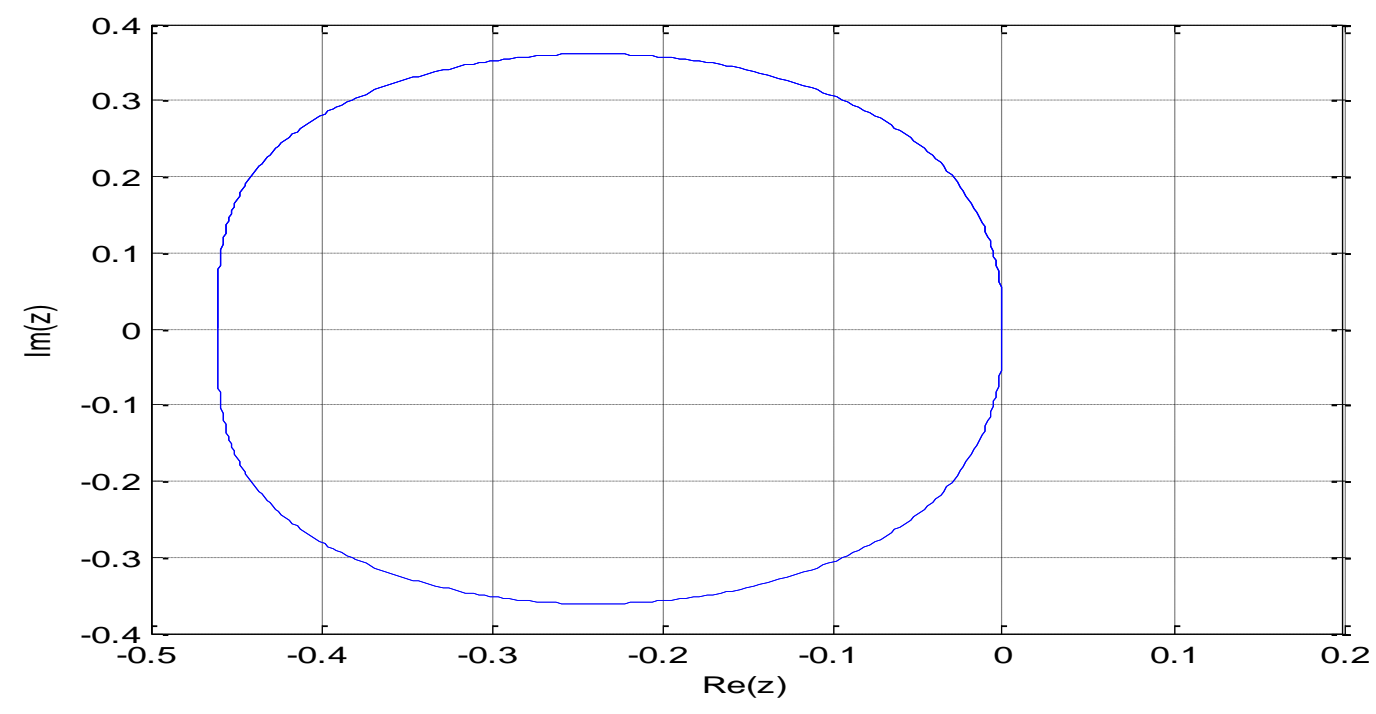

\subsection{Numerical Example}

Problem I. We consider a highly stiff problem $y^{\prime \prime}+1001 y^{\prime}+1000 y, \quad y(0)=1, y^{\prime}(0)=-1$ 
Exact Solution: $y(x)=\exp (-x) h=\frac{1}{10}$

Table-1. Comparison of the proposed method with Adeniran and Ogundare (2015)

\begin{tabular}{l|l|l|l|l}
\hline $\begin{array}{l}\text { x- } \\
\text { values }\end{array}$ & Exact Solution & Computed Solution & $\begin{array}{l}\text { Error in our } \\
\text { method }\end{array}$ & $\begin{array}{l}\text { Error in Adeniran } \\
\text { \& Ogundare (2015) }\end{array}$ \\
\hline 0.100 & 0.90483741803595957316 & 0.90483741803595958558 & $1.24200 \mathrm{E}(-17)$ & $2.05 \mathrm{E}(-11)$ \\
\hline 0.200 & 0.81873075307798185867 & 0.81873075307798185409 & $4.58000 \mathrm{E}(-18)$ & $4.39 \mathrm{E}(-11)$ \\
\hline 0.300 & 0.74081822068171786607 & 0.74081822068171801968 & $1.53610 \mathrm{E}(-16)$ & $6.55 \mathrm{E}(-11)$ \\
\hline 0.400 & 0.67032004603563930074 & 0.67032004603563944601 & $1.45270 \mathrm{E}(-16)$ & $8.38 \mathrm{E}(-11)$ \\
\hline 0.500 & 0.60653065971263342360 & 0.60653065971263356194 & $1.38340 \mathrm{E}(-16)$ & $9.86 \mathrm{E}(-11)$ \\
\hline 0.600 & 0.54881163609402643263 & 0.54881163609402656540 & $1.32770 \mathrm{E}(-16)$ & $1.10 \mathrm{E}(-10)$ \\
\hline 0.700 & 0.49658530379140951470 & 0.49658530379140964328 & $1.28580 \mathrm{E}(-16)$ & $1.19 \mathrm{E}(-10)$ \\
\hline 0.800 & 0.44932896411722159143 & 0.44932896411722171709 & $1.25660 \mathrm{E}(-16)$ & $1.24 \mathrm{E}(-10)$ \\
\hline 0.900 & 0.40656965974059911188 & 0.40656965974059923585 & $1.23970 \mathrm{E}(-16)$ & $1.28 \mathrm{E}(-10)$ \\
\hline 1.00 & 0.36787944117144232160 & 0.36787944117144244514 & $1.23540 \mathrm{E}(-16)$ & $1.30 \mathrm{E}(-10)$ \\
\hline
\end{tabular}

Problem II. We consider the second order ODE

Exact Solution:

$$
\begin{aligned}
& \qquad f\left(x, y, y^{\prime}\right)=100 y, \quad y(0)=1, y^{\prime}(0)=-10 . \\
& y(x)=e^{-10 x} \text { with } h=\frac{1}{100}
\end{aligned}
$$

Table-2. Comparison of the proposed method with Mohammad and Zurni [3]

\begin{tabular}{l|l|l|l|l}
\hline $\begin{array}{l}\text { x- } \\
\text { values }\end{array}$ & Exact Solution & Computed Solution & $\begin{array}{l}\text { Error in our } \\
\text { method }\end{array}$ & $\begin{array}{l}\text { Error in Mohammad } \\
\text { and Zurni [3] }\end{array}$ \\
\hline 0.01 & 0.90483741803595957316 & 0.90483741803595957318 & $2.0000 \mathrm{E}(-20)$ & 0.0000000000 \\
\hline 0.02 & 0.81873075307798185867 & 0.81873075307798185869 & $2.0000 \mathrm{E}(-20)$ & $2.431388 \mathrm{E}(-14)$ \\
\hline 0.03 & 0.74081822068171786607 & 0.74081822068171786605 & $2.0000 \mathrm{E}(-20)$ & $7.105427 \mathrm{E}(-14)$ \\
\hline 0.04 & 0.67032004603563930074 & 0.67032004603563930074 & 0.000000000 & $1.384448 \mathrm{E}(-13)$ \\
\hline 0.05 & 0.60653065971263342360 & 0.60653065971263342358 & $2.0000 \mathrm{E}(-20)$ & $2.257083 \mathrm{E}(-13)$ \\
\hline 0.06 & 0.54881163609402643263 & 0.54881163609402643258 & $5.0000 \mathrm{E}(-20)$ & $3.316236 \mathrm{E}(-13)$ \\
\hline 0.07 & 0.49658530379140951470 & 0.49658530379140951466 & $4.0000 \mathrm{E}(-20)$ & $4.555800 \mathrm{E}(-13)$ \\
\hline 0.08 & 0.44932896411722159143 & 0.44932896411722159138 & $5.0000 \mathrm{E}(-20)$ & $5.974665 \mathrm{E}(-13)$ \\
\hline 0.09 & 0.40656965974059911188 & 0.40656965974059911180 & $8.0000 \mathrm{E}(-20)$ & $7.575052 \mathrm{E}(-13)$ \\
\hline 0.10 & 0.36787944117144232160 & 0.36787944117144232150 & $1.0000 \mathrm{E}(-19)$ & $8.361956 \mathrm{E}(-13)$ \\
\hline 0.11 & 0.33287108369807955329 & 0.33287108369807955318 & $1.1000 \mathrm{E}(-19)$ & $1.134096 \mathrm{E}(-19)$ \\
\hline 0.12 & 0.30119421191220209664 & 0.30119421191220209651 & $1.3000 \mathrm{E}(-19)$ & $1.352474 \mathrm{E}(-12)$ \\
\hline
\end{tabular}

Problem III. $f\left(x, y, y^{\prime}\right)=y^{\prime}, \quad y(0)=1, y^{\prime}(0)=-1,0 \leq x \leq 1$.

Exact Solution: $y(x)=1-e^{x}$ with $h=\frac{1}{10}$

Table-3. Comparison of the proposed method with Raymond, et al. [1]

\begin{tabular}{l|l|l|l|l}
\hline $\begin{array}{l}\mathbf{x}- \\
\text { values }\end{array}$ & Exact Solution & Computed Solution & $\begin{array}{l}\text { Error in our } \\
\text { method }\end{array}$ & $\begin{array}{l}\text { Error in Raymond, et } \\
\text { al. }[\mathbf{1}]\end{array}$ \\
\hline 0.1 & -0.1051709180756476248 & -0.10517091807564762482 & $2.0000 \mathrm{E}(-20)$ & $1.0000 \mathrm{E}(-20)$ \\
\hline 0.2 & -0.2214027581601698339 & -0.22140275816016983392 & $2.0000 \mathrm{E}(-20)$ & $2.0000 \mathrm{E}(-20)$ \\
\hline 0.3 & -0.3498588075760031040 & -0.34985880757600310399 & $1.0000 \mathrm{E}(-20)$ & $3.9000 \mathrm{E}(-19)$ \\
\hline 0.4 & -0.4918246976412703178 & -0.49182469764127031785 & $5.0000 \mathrm{E}(-20)$ & $1.1600 \mathrm{E}(-18)$ \\
\hline 0.5 & -0.6487212707001281468 & -0.64872127070012814688 & $8.0000 \mathrm{E}(-20)$ & $2.0700 \mathrm{E}(-18)$ \\
\hline 0.6 & -0.8221188003905089749 & -0.82211880039050897492 & $2.0000 \mathrm{E}(-20)$ & $3.3900 \mathrm{E}(-18)$ \\
\hline 0.7 & -1.0137527074704765216 & -1.01375270747047652170 & $1.0000 \mathrm{E}(-19)$ & $5.8000 \mathrm{E}(-18)$ \\
\hline 0.8 & -1.2255409284924676046 & -1.22554092849246760470 & $1.0000 \mathrm{E}(-19)$ & $8.3000 \mathrm{E}(-18)$ \\
\hline 0.9 & -1.4596031111569496638 & -1.45960311115694966390 & $1.0000 \mathrm{E}(-19)$ & $1.15000 \mathrm{E}(-17)$ \\
\hline 1.0 & -1.7182818284590452354 & -1.71828182845904523550 & $1.0000 \mathrm{E}(-19)$ & $1.60000 \mathrm{E}(-17)$ \\
\hline
\end{tabular}

\section{Conclusions}

It is shown from the tables of result that our proposed methods are indeed accurate, and can handle stiff equations. Comparing the new method with the existing method of Adeniran and Ogundare [5], Mohammad and Zurni [3] and Raymond, et al. [2],the result presented in the tables 1, 2 and 3 respectively shows that the new method 
performs better than the existing method. Three steps with two offgrid points method is derive through collocation and interpolation technique, the developed method converges and is of Order twelve.

\section{References}

[1] Raymond, D., Skwame, Y., and Joshua, S., 2018. "An implicit two-step one off-grid point third derivative hybrid block method for the direct solution of second order ordinary differential equations." Academic Journal of Applied Mathematical Science, vol. 4, pp. 8-14.

[2] Raymond, D., Skwame, Y., and Kyagya, S. T., 2018. "An efficient third derivative hybrid block method for the direct solution of second order ordinary differential equations." Asian Research Journal of Mathematics, vol. 9, pp. 1-10.

[3] Mohammad, A. and Zurni, O., 2017. "Generalized two-hybrid one-step implicit third derivatives block method for the direct solution of second order ordinary differential equations." International Journal of Pure and Applied Mathematics, vol. 112, pp. 497-517.

[4] Abdelrahim, R. and Zurni, O., 2016. "Direct solution of second-order ordinary differential equation using a single-step hybrid block method of order five." Mathematical and Computational Applications, vol. 21, p. 12.

[5] Adeniran, A. O. and Ogundare, B. S., 2015. "An efficient hybrid numerical scheme for solving general second order initial value problems (IVPs)." International Journal of Applied Mathematical Research, vol. 4, pp. 411-419.

[6] Adesanya, A. O., Alkali, M. A., and Sunday, J., 2014. "Order five hybrid block method for the solution of second order ordinary differential equation." International J. of Math. Sci. and Engg. Appls, vol. 8, pp. 285295.

[7] Awoyemi, D. O., Adesanya, A. O., and Ogunyebi, S. N., 2009. "Construction of self-starting numerov method for the solution of initial value problem of general second order ordinary differential equation." Journ. Num. Math, vol. 4, pp. 267-278.

[8] Fatunla, S. O., 1991. "Block methods for second order IVPs." Int. J. Comput. Maths., pp. 4155-63. 